\title{
DE LA COLÈRE A LA SOUMISSION DANS LES CONTEMPLATIONS DE VICTOR HUGO
}

\section{FROM ANGER TO SUBMISSION IN VICTOR HUGO'S LES CONTEMPLATIONS}

AMIR BIGLARI*

RÉSUMÉ : Cette étude se propose d'analyser deux états passionnels successifs dans Les Contemplations de Victor Hugo : d'abord la colère ("Trois ans après ») et ensuite la soumission (« À Villequier »). En se référant à la théorie sémiotique, elle tentera d'élucider les caractéristiques discursives de ces deux états. Plus précisément, en faisant appel au schéma passionnel canonique, au point de vue tensif et à la sémiotique des instances, elle mettra l'accent sur les transformations actantielles, modales, spatiales, temporelles, aspectuelles, axiologiques et énonciatives apparues lors de ce passage. Parallèlement, ce travail s'attachera à mettre l'efficacité heuristique de la théorie sémiotique à l'épreuve du texte.

MOTS-CLÉS : Colère. Soumission. Victor Hugo. Sémiotique. Tensivité.

* Chercheur associé au CeReS-UNILIM - Centre de Recherches Sémiotiques de l'Université de Limoges. E-mail: biglari_amir82@yahoo.fr. 
ABSTRACT: This study aims to analyze two successive emotional states in Victor Hugo's Les Contemplations: first that of anger ("Trois ans après"), followed by that of submisson ("A Villequier"). An attempt is made to apply the semiotic theory in order to elucidate the discursive characteristics of these states. Specifically, by using the canonic emotional scheme, the tensive point of view and the semiotics of instances, this analysis highlights the actantial, modal, spatial, temporal, aspectual, axiological and enunciative transformations taking place during this change. At the same time, this work intends to test the heuristic efficiency of the semiotic theory.

KEYWORDS : Anger. Submission. Victor Hugo. Semiotics. Tensivity.

Les Contemplations de Victor Hugo est l'un des chefsd'œuvre du romantisme, à savoir le mouvement littéraire qui a su rétablir la passion, souvent considérée comme de « mauvaise réputation » (COLLOT, 1997, p. 09) ${ }^{1}$ : «Le romantisme retrouve un sens intentionnel positif à la passion, qui devient le ressort indispensable et enthousiasmant de l'action créatrice » (RALLO DITCHE ; FONTANILLE, 2005, p. 08).

Ce recueil de poèmes constitue une véritable exposition où les passions les plus variées occupent le devant de la

1 Il explique : «L'émotion a mauvaise réputation. Collective, elle se prête à toutes sortes de débordements et de manipulations, au profit parfois des idéologies les plus dangereuses. Individuelle, elle implique une perte de maîtrise, une aliénation de soi et de l'autre. Nuisible politiquement et moralement, elle est désastreuse en poésie. L'expression sans retenue des émotions, croyant atteindre la singularité et l'authenticité, reproduit en général les pires stéréotypes, faute d'avoir soumis ce qui est vécu et ressenti à la moindre élaboration » (COLLOT, 1997, p. 09). 
scène. Ici, nous nous intéressons à la colère, passion qui s'y manifeste brièvement et localement : le locuteur se met en colère et se révolte contre Dieu dans "Trois ans après ", le troisième poème du quatrième livre ${ }^{2}$. Plus tard, dans le quinzième poème du même livre, «À Villequier », le locuteur, cette fois soumis, donne des explications à propos de son état passionnel apparu dans « Trois ans après », et en réhabilitant Dieu, tente de se justifier.

Le présent travail propose une description de ces deux états passionnels en ayant recours à la théorie sémiotique, notamment à la sémiotique des passions, à la sémiotique tensive et à la sémiotique des instances. Il essaiera ainsi d'apporter un éclairage sur les particularités de la mise en discours hugolienne, en mettant en relief les enjeux qui se dégagent de cette transformation passionnelle. Chemin faisant, il s'agit d'éprouver la valeur analytique de la théorie sémiotique.

\section{De la colère}

Commençons par citer les treize strophes de « Trois ans après », où le locuteur manifeste sa colère :

L'humble enfant que Dieu m'a ravie

Rien qu'en m'aimant savait m'aider ;

C'était le bonheur de ma vie

De voir ses yeux me regarder.

2 Une précision terminologique s'impose. Bien que particulièrement proches, la colère et la révolte sont deux états distincts : en général, la révolte implique une remise en question des systèmes de valeurs et du destinateur, ce qui n'est pas le cas de la colère dans ses limites propres (FONTANILLE, 2005, p. 61-69). Pourtant, compte tenu des enchevêtrements sémantiques et syntaxiques entre ces deux passions, particulièrement dans « Trois ans après ", où il s'agit en réalité d'une colère révoltée, nous utiliserons librement les termes de « colère » ou de « révolte » pour désigner l'état du locuteur du poème. 
Si ce Dieu n'a pas voulu clore L'œuvre qu'il me fit commencer, S'il veut que je travaille encore, Il n'avait qu'à me la laisser !

Il n'avait qu'à me laisser vivre Avec ma fille à mes côtés, Dans cette extase où je m'enivre De mystérieuses clartés !

Ces clartés, jour d'une autre sphère, Ô Dieu jaloux, tu nous les vends ! Pourquoi m'as-tu pris la lumière Que j'avais parmi les vivants?

As-tu donc pensé, fatal maître, Qu'à force de te contempler, Je ne voyais plus ce doux être, Et qu'il pouvait bien s'en aller?

T'es-tu dit que l'homme, vaine ombre, Hélas ! perd son humanité À trop voir cette splendeur sombre Qu'on appelle la vérité?

Qu'on peut le frapper sans qu'il souffre, Que son cœur est mort dans l'ennui, Et qu'à force de voir le gouffre, Il n'a plus qu'un abîme en lui ?

Qu'il va, stoïque, où tu l'envoies, Et que désormais, endurci, N'ayant plus ici-bas de joies, Il n'a plus de douleurs aussi ?

As-tu pensé qu'une âme tendre S'ouvre à toi pour mieux se fermer, 
Et que ceux qui veulent comprendre

Finissent par ne plus aimer?

Ô Dieu ! vraiment, as-tu pu croire

Que je préférais, sous les cieux,

L'effrayant rayon de ta gloire

Aux douces lueurs de ses yeux?

Si j'avais su tes lois moroses,

Et qu'au même esprit enchanté

Tu ne donnes point ces deux choses,

Le bonheur et la vérité,

Plutôt que de lever tes voiles,

Et de chercher, cœur triste et pur,

À te voir au fond des étoiles,

Ô Dieu sombre d'un monde obscur,

J'eusse aimé mieux, loin de ta face,

Suivre, heureux, un étroit chemin,

Et n'être qu'un homme qui passe

Tenant son enfant par la main! (HUGO, 2008, p. 196-197)33.

Il s'agit en effet, en termes actantiels, d'une relation polémique entre un sujet et son destinateur, à savoir l'«instance d'autorité » (BERTRAND, 2000, p. 228) « qui définit l'ordre des valeurs en jeu » (BERTRAND, 2000, p. 261), le " siège d'un pouvoir transcendant et irréversible » (COQUET, 1989 [1984], p. 10, c'est l'auteur qui souligne) : le locuteur se révolte contre le Dieu biblique - Ancien Testament - qui règle la vie de l'homme. C'est la rupture du contrat fiduciaire par le destinateur et la sanction négative reçue qui font se révolter le sujet contre le destinateur (plus précisément,

3 Nous nous référons à l'édition de Pierre Laforgue, publiée en 2008. 
l'anti-destinateur) : " on doute de lui, on s'interroge sur ses intentions, on finit par lui dénier toute compétence. Il est virtualisé par le faire interprétatif de sujet » (FONTANILLE, 1980, p. 08).

Le passage s'ouvre sur la mise en accusation de Dieu : celui-ci a « ravi » l'enfant du locuteur. « Ravir » est défini par le dictionnaire Littré comme « Enlever de force, par violence »; « Ôter, priver de ». L'acte de Dieu, ayant pour conséquence la privation du sujet de son objet de valeur, et d'ailleurs accompagné de «force » et de « violence », montre sa mauvaise foi et implique la tension. L'objet de valeur est évoqué dans le contexte d'un passé heureux, et son savoir faire ainsi que son pouvoir faire sont mis en valeur (« Rien qu'en m'aimant savait m'aider »). La deuxième et la troisième strophes insistent sur la divergence aspectuelle qui correspond à une confrontation modale : le programme d'abrègement aspectuel du locuteur s'oppose au programme d'allongement aspectuel de Dieu, et le locuteur est obligé de subir deux devoirs (ne pas pouvoir ne pas faire) : laisser sa fille partir et continuer à vivre. S'il a dû subir le premier devoir, se trouvant donc dans un monde sans le véritable objet de valeur, il désire, au moins, ne pas subir le deuxième. En réalité, l'attitude de Dieu est dénoncée comme inconséquente, comme s'il ne savait pas ce qu'il voulait : on est au bord du blasphème. Autant l'image de la fille est méliorative, autant la figure de Dieu est péjorative. Le locuteur actualise un marchandage, un échange qui n'a pas eu lieu : Dieu aurait pu laisser la fille, et de son côté le locuteur aurait poursuivi son œuvre. La répétition de la formule «ne ... que » renforce la focalisation péjorative.

L'isotopie de la péjoration de Dieu se prolonge dans la strophe suivante avec le verbe "vendre » et le verbe " prendre », dans une formulation qui évoque le modèle de 
l'interrogation judiciaire face à un voleur. Par ailleurs, Dieu est considéré comme " jaloux », c'est-à-dire qu'au plan du faire, il contredit sa nature qui est la bonté, il garde pour soi au lieu de donner. Le locuteur attribue à Dieu le même statut actantiel que lui-même, il le réduit du statut du destinateur à celui de l'anti-sujet : à la jalousie, la rivalité est intrinsèque, et celle-ci n'est possible qu'entre deux actants du même statut autour d'un seul objet de valeur. Cette qualification met également l'accent sur l'importance de l'objet de valeur : même Dieu refuse de le partager, il veut l'avoir en exclusivité 4

Par ailleurs, dans cette strophe, un changement énonciatif essentiel, maintenu jusqu'à la fin, se fait jour : Dieu qui était en dehors de la scène d'énonciation, y entre soudainement. Cette modification de mode d'énonciation est d'autant plus importante que «Dieu » est presque toujours, au cours du recueil, un « il »- contrairement au mot « Seigneur», qui apparaît souvent en situation d'énonciation ${ }^{5}$. Sa présence sur la scène d'énonciation est donc rare, et s'il s'y manifeste, il est toujours accompagné d'un adjectif, tantôt en vouvoiement, tantôt en tutoiement. Par exemple : «Dieu tout-puissant » (« Lettre », p. 87), « Dieu sévère » (« Claire P. », p. 252), « Dieu serein » («Pleurs dans la nuit», p. 294) en vouvoiement; « Grand Dieu » (« La vie aux champs », p. 36) en tutoiement, dans une situation d'éloge. Mais, dans le passage que nous

4 Dans le poème qui précède « Trois ans après », à savoir «Aime celui qui t'aime... », la même situation, susceptible de produire la jalousie, se fait jour : un autre actant aime sa fille, et veut se l'approprier. Mais la jalousie est tout à fait absente, car le sujet est soumis au faire devoir imposé : «Aime celui qui t'aime, et sois heureuse en lui. / - Adieu ! - Sois son trésor, ô toi qui fus le nôtre ! / Va, mon enfant béni, d'une famille à l'autre. / Emporte le bonheur et laisse-nous l'ennui ! / Ici, l'on te retient ; là-bas, on te désire. / Fille, épouse, ange, enfant, fais ton double devoir. / Donne-nous un regret, donne-leur un espoir, / Sors avec une larme ! entre avec un sourire!» (p. 194).

5 On peut visiblement observer ce contraste énonciatif entre les termes « Dieu » et « Seigneur » dans le vers suivant, valable pour le recueil entier : « J'ai dit à Dieu : "Seigneur, jugez où nous en sommes [“] » («Écoutez. Je suis Jean. J'ai vu des choses sombres », p. 284). 
venons de citer, le tutoiement est utilisé dans une situation de reproche : «Dieu jaloux ». On peut montrer ces évolutions énonciatives sur un schéma tensif ${ }^{6}$ :

Schéma 1 - Evolutions Enonciatives.

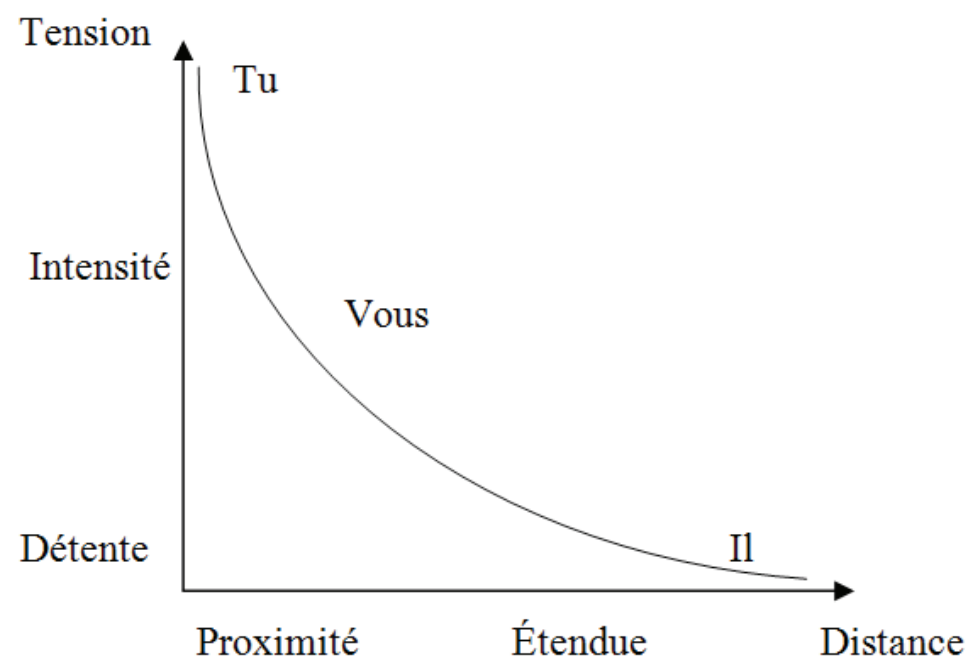

Le passage $\mathrm{au}$ « tu » produit donc ici un effet de rapprochement, defaceàface, d'accélération etd'augmentation de la tension. Cette transformation énonciative du « il » au « tu » correspond au changement d'étape selon la séquence canonique de la colère ${ }^{7}$ : confiance $\rightarrow$ attente $\rightarrow$ frustration $\rightarrow$ mécontentement $\rightarrow$ agressivité $\rightarrow$ explosion $^{8}$.

6 Pour une présentation de la sémiotique tensive, voir par exemple : Zilberberg (2006, 2012).

7 Voir Fontanille (2005, p. 63).

8 Cette séquence canonique est « fondée sur une chaîne de «raisons» : le sujet «explose» en raison de son agressivité ; il est agressif «en raison» de son mécontentement, il est mécontent «en raison» de sa déception, il est déçu «en raison» de ce qu'il attendait, [...] il attendait «en raison» de ce qu'on lui avait promis ou laissé espérer » (FONTANILLE, 2005, p. 63). 
En réalité, de façon générale, le sujet de colère est un sujet qui attendait à la suite de ce qu'on lui avait promis ou laissé espérer, mais dont l'attente est déçue ; d'où sa " frustration » dans un premier temps, phase qui émerge lorsque le sujet éprouve le manque «sur le fond de la confiance et de l'attente déçues », lorsqu'il " réactualise la promesse de conjonction antérieure » (FONTANILLE, 2005, p. 64). En l'occurrence, cette phase apparaît dans les strophes qui précèdent celles que nous avons citées : «[...] je souffre comme père, Moi qui souffris tant comme enfant! » (HUGO, 2008, p. 195), etc. Dans cette phase, le locuteur n'évoque pas Dieu, en revanche, à la phase du "mécontentement », Dieu se manifeste sous forme débrayée, et aux moments de l'« agressivité » et de l'« explosion», sous forme adressée.

Dans la phase du mécontentement, « le sujet confronte ce qu'il espérait et ce qu'il obtient (l'état attendu et l'état réalisé), et conclut à une situation insatisfaisante, à une inadéquation entre le soi projeté et le moi actuel » (FONTANILLE, 2005, p. 65) : «L'humble enfant que Dieu m’a ravie / Rien qu'en m'aimant savait m'aider ; / C'était le bonheur de ma vie / De voir ses yeux me regarder », etc. Quant à l'« agressivité », elle « est explicitement adressée à cet autre sujet, le fautif, le traître, celui qui n'a pas honoré sa promesse » (FONTANILLE, 2005, p. 64). Cette phase commence avec la quatrième strophe, en même temps que Dieu entre sur la scène d'énonciation, et se confond au fur et à mesure avec l'« explosion », difficile à distinguer nettement de l'« agressivité », qui apparaît plutôt dans les dernières strophes que nous avons citées. Nous y reviendrons.

Dans la cinquième strophe, la tension augmente : après avoir été considéré comme " jaloux », Dieu est ici considéré 
comme le " fatal maître », le cruel qui fait mourir". Cette succession est ascendante : le « fatal maître » est plus proche et plus menaçant que « Dieu jaloux ».

Cette strophe, ainsi que celles qui la suivent, mettent en scène deux paradigmes : d'une part, il s'agit de la contemplation, de la recherche de la vérité, de la compréhension ; de l'autre, de la sensibilité, de l'humanité, de l'amour et du bonheur. Le locuteur avait cru à la pertinence de l'opération de mélange ${ }^{10}$, il avait estimé que ces grandeurs étaient conciliables (et... et...). Ce n'est que maintenant, après avoir perdu sa fille, qu'il apprend que ce n'est pas le cas ("Si j'avais su tes lois moroses ») : le locuteur affirme que Dieu impose une opération de tri, en jugeant que ces deux paradigmes sont incompatibles, qu'ils sont exclusifs (ou... ou...) : la compréhension exclut l'amour (strophe 9) ; la vérité s'oppose au bonheur (strophe 11) ; plus la contemplation et la recherche de la vérité durent, plus elles déshumanisent et insensibilisent (strophes 5-8). Soit le schéma suivant:

9 Si l'on peut dire avec Ludmila Charles-Wurtz que c'est « souvent aux femmes que Hugo attribue le discours le plus critique envers Dieu », que les « mères en deuil se révoltent ouvertement contre Dieu, lui demandant des comptes » (HUGO, 1998, p. 227), ce n'est pas le cas ici. Dans cette occurrence très critique et blasphématoire, peut-être la plus forte dans l'œuvre de Hugo, la parole est assumée par un acteur masculin, d'ailleurs en « je ». De plus, l'image d'un Dieu négatif se trouve aussi dans d'autres ouvrages de Hugo. Il note par exemple dans Les Misérables: « Je ne comprends pas comment Dieu, le père des hommes, peut torturer ses enfants et ses petits-enfants et les entendre crier sans être torturé lui-même » (HUGO, 1998 [1862], p. 1346).

10 Le «mélange » et le «tri » sont les deux grandes opérations de la « syntaxe extensive » (ZILBERBERG, 2012, p. 151 et p. 159). 
Schéma 2 - Point de vue attribué à Dieu.

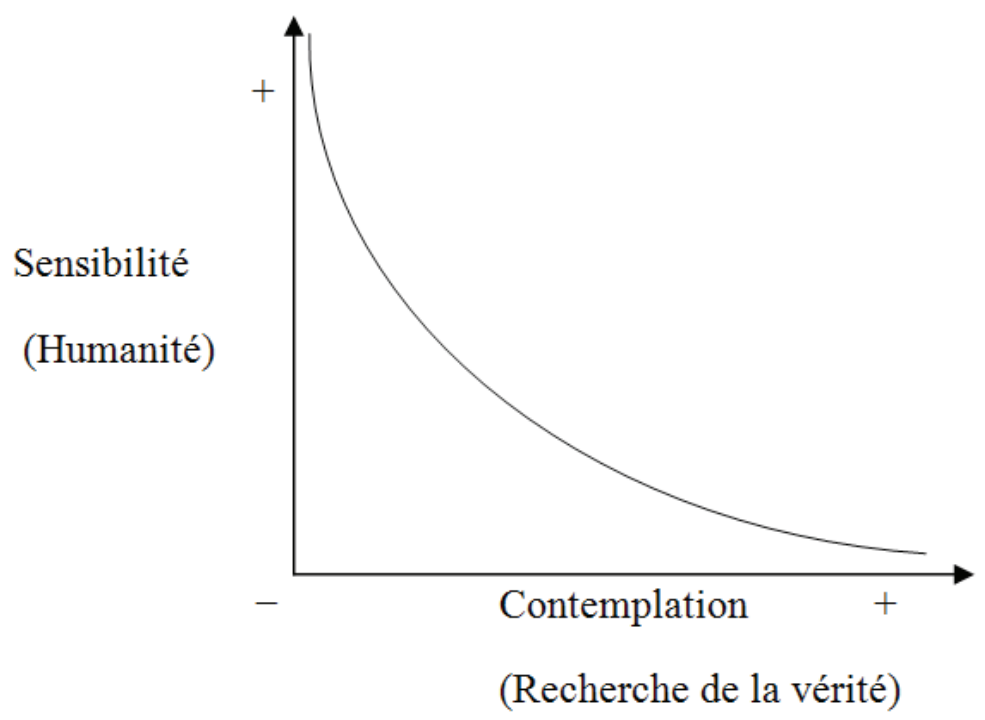

De la cinquième à la neuvième strophe, le ton reste identique : des questions commençant par des expressions comme «As-tu donc pensé », "T'es-tu dit », « As-tu pensé », remettent en cause le raisonnement attribué à Dieu. Dans la dixième strophe, le ton se durcit : « as-tu pu croire », qui est une formule plus sévère, est accentué par un adverbe intensificateur ("vraiment »), ainsi que par une apostrophe («Ô Dieu !») ; de plus, à travers une antithèse, fondée sur l'isotopie de la lumière, le locuteur met en valeur sa fille et méprise Dieu : «L'effrayant rayon de ta gloire » vs « douces lueurs de ses yeux».

Le ton plus ferme de la dixième strophe semble favoriser le passage aux trois dernières strophes, où le ton est le plus intense, dans la mesure où l'adresse à Dieu sous forme d'interrogation cède la place à l'affirmation directe du locuteur, selon laquelle il opte pour sa fille au détriment de 
Dieu. De nouvelles antithèses se font jour : si le paradigme de Dieu a pour horizon le « fond des étoiles», et s'il a affaire aux valeurs visuelles ( te voir ») et à la distance ( loin de ta face »), celui de sa fille a pour horizon « un étroit chemin », il a trait aux valeurs tactiles (« Tenant son enfant par la main ») et à la proximité (présupposée par « tenir par la main »).

L'ensemble des strophes explorent, en effet, deux microunivers qui s'opposent sur plusieurs plans : (i) celui qui a pour fin la contemplation, qui relève donc du régime discursif de la cognition, et qui a pour corrélat le logos ; (ii) celui qui a pour fin la paternité, qui relève du régime discursif de la passion et de la sensibilité, et qui a pour corrélat le phusis $^{11}$ :

Tableau 1 - Deux micro-univers.

\begin{tabular}{|l|l|l|}
\hline & La contemplation & La paternité \\
\hline Régime discursif dominant & Cognition & Passion (sensibilité) \\
\hline Instance impliquée & Logos & Phusis \\
\hline Programme & Compréhension & Amour \\
\hline Objet assorti & Vérité & Bonheur \\
\hline Valeurs sensibles & Valeurs visuelles & Valeurs tactiles \\
\hline Fiducie & Défiance, inquiétude & Confiance \\
\hline Spatialité & Distance considérable & Proximité, contact \\
\hline Horizon & Fond des étoiles & Étroit chemin \\
\hline
\end{tabular}

Comme on l'a dit, selon la logique de tri attribuée à Dieu, le choix de l'un des paradigmes implique le renoncement à l'autre, ce qui va à l'encontre de l'attente du locuteur, qui avait cru à la solidarité des deux paradigmes, à un schéma tensif converse et non pas inverse. En effet, la raison de la profonde 
insatisfaction du locuteur, outre la perte de son objet de valeur, c'est que le destinateur n'a pas respecté le contrat fiduciaire : le locuteur se révolte dans la mesure où le système de valeurs qui avait fondé sa confiance en le destinateur n'est plus considéré comme valable pour celui-ci. Aussi, étant dans l'obligation de faire un choix entre les deux paradigmes, le locuteur remet en cause le paradigme directement lié au destinateur, celui de la divinité, et opte pour la paternité.

Par ailleurs, les trois dernières strophes citées coïncident avec la phase de l'« explosion »sur la séquence canonique, celle qui " réunit en somme toutes les identités modales et affectives traversées au cours de la séquence en une manifestation unique, massive et sans étendue » (FONTANILLE, 2005, p. 65), qui, en l'occurrence, voit le jour avec l'adresse directe au destinateur, la remise en cause de celui-ci et de son système de valeurs, ainsi que la sélection du paradigme qui lui est opposé.

Après l'analyse des strophes de "Trois ans après » centrées sur la colère et la révolte contre $\mathrm{Dieu}^{12}$, nous allons étudier le poème « À Villequier » qui y répond.

12 Il convient d'ajouter que la colère et la révolte sont des motifs fréquents chez les romantiques. Elles peuvent être de différentes natures : ontologiques, sociales, politiques, etc., et vont en général avec un individualisme hostile et un esprit de négation (un exemple célèbre se trouve dans La Coupe et les lèvres d'Alfred de Musset : 2007 [1831], p. 26). Cela fait écho au « beau geste » défini par Greimas et Fontanille (1993, p. 34), « une invention par négation », qui se rattache d'ailleurs ici à une forme de vie à part entière. Ce n'est donc pas étonnant que les romantiques soient fascinés par Satan, le révolté suprême contre Dieu. Parmi les incarnations les plus parlantes de cet esprit de négation, on peut surtout mentionner la figure de Satan dans La Fin de Satan (1886) de Hugo, et celle de Méphistophélès, l'esprit qui toujours nie, dans Faust (1808) de Goethe. C'est dans la même perspective que Hugo prend Dieu pour le « fatal maître » ou « Dieu jaloux » dans Les Contemplations. Cette deuxième expression, « Dieu jaloux », est aussi utilisée dans Les Châtiments (1967 [1853], « Cette nuit, il pleuvait, la marée était haute », p. 199), ou bien dans les Poèmes antiques et modernes de Vigny (2007 [1826], « Moïse », p. 24). De la même manière, Pétrus Borel prend Dieu, dans ses Rhapsodies, pour un « ogre appelé Dieu » (2009 [1832], « Rêveries », p. 76). Dieu semble donc être l'une des principales cibles de la colère romantique, car considéré comme l'anti-destinateur, celui qui a promis ou qui a fait espérer sans avoir respecté son engagement, celui qui a unilatéralement rompu le contrat fiduciaire. 


\section{De la soumission}

Le locuteur de «À Villequier » donne des explications à propos de sa révolte contre Dieu, et tente de se justifier auprès de lui. Il est toujours sombre et un peu amer, mais « attendri » (p. 211) et « éclairé » (p. 214) ; il se soumet à l'ordre divin. Nous nous focalisons, en trois temps, sur les passages qui ont un rapport direct avec la colère révolue.

\section{Du non-sujet au sujet}

Maintenant que Paris, ses pavés et ses marbres, Et sa brume et ses toits sont bien loin de mes yeux; Maintenant que je suis sous les branches des arbres, Et que je puis songer à la beauté des cieux ;

Maintenant que du deuil qui m'a fait l'âme obscure

Je sors, pâle et vainqueur,

Et que je sens la paix de la grande nature

Qui m'entre dans le cœur ;

Maintenant que je puis, assis au bord des ondes, Ému par ce superbe et tranquille horizon, Examiner en moi les vérités profondes

Et regarder les fleurs qui sont dans le gazon ;

Maintenant, ô mon Dieu ! que j'ai ce calme sombre De pouvoir désormais

Voir de mes yeux la pierre où je sais que dans l'ombre Elle dort pour jamais ;

Maintenant qu'attendri par ces divins spectacles, Plaines, forêts, rochers, vallons, fleuve argenté, Voyant ma petitesse et voyant vos miracles, Je reprends ma raison devant l'immensité ; [...] (HUGO, 2008, p. 210-211). 
Commençons par la fin. Dans le dernier vers, le locuteur dit qu'il « reprend sa raison ». S'il la « reprend », c'est qu'il l'avait perdue au moment de la révolte, qu'il avait donc perdu son contrôle et sa capacité de jugement, qu'il était devenu non-sujet ${ }^{13}$. Rappelons par ailleurs que le poème qui suit "Trois ans après », c'est "Oh! je fus comme fou dans le premier moment », poème qui souligne qu'au moment de la disparition de sa fille, vu le caractère tonique et inattendu de l'événement, le locuteur était devenu non-sujet. Il reste maintenant à savoir pourquoi il se retransforme de non-sujet en sujet, comment s'effectue la conversion d'un actant particulièrement passionnel à un actant chez qui le cognitif tend à dominer le passionnel. Trois paramètres sont mentionnés dans les strophes supra: (i) le passage du temps ; (ii) son rapprochement de la tombe de sa fille; (iii) l'influence de la nature. Examinons-les.

Le passage du temps est surtout indiqué par l'expression répétée "maintenant que », formulation qui, ici, signale non seulement une rupture d'isotopie temporelle par rapport à un alors, mais aussi une rupture par rapport à un état d'âme antérieur. Le participe passé " attendri » va dans le même sens, car, étant une modalité du devenir, il correspond à une transformation d'état d'âme.

De plus, la quatrième strophe, ainsi que le titre même du poème, «À Villequier », indiquent que le locuteur n'est plus en exil, mais à côté de la tombe de sa fille : «pouvoir désormais / Voir de mes yeux la pierre où je sais que dans l'ombre / Elle

13 Voir par exemple : Coquet (1997, p. 01-18); Coquet (2007, p. 121-134, p. 252-262). Le sujet correspond à l'instance judicative, celle qui pense, qui assume son énonciation, qui est un actant-source ; en revanche, le non-sujet correspond à l'instance corporelle, celle qui perçoit, qui prédique sans assumer, qui est un actant-cible. Envahi par une force passionnelle, le nonsujet est réduit à une simple présence au monde, dépourvu de jugement et privé de toute modalité. 
dort pour jamais ». Il est à noter que ce poème est placé juste après le poème «Demain, dès l'aube... », et daté du lendemain de celui-ci, comme si « À Villequier » était la réalisation de la décision de la veille. Sa présence sur la tombe de sa fille est à l'origine de " ce sombre calme », ce qui montre qu'un "schéma de l'atténuation » est activé, c'est-à-dire un schéma dont l'« abaissement de l'intensité conjugué à la réduction de l'étendue procure une détente générale » (FONTANILLE, 2003, p. 112) ; si pendant l'exil, l'éloignement de la tombe de sa fille faisait écho à la tension (dysphorie tendue), à présent le rapprochement de la tombe va avec la détente (dysphorie atone) du sujet :

Schéma 3 - De l'éxil à la tombe.

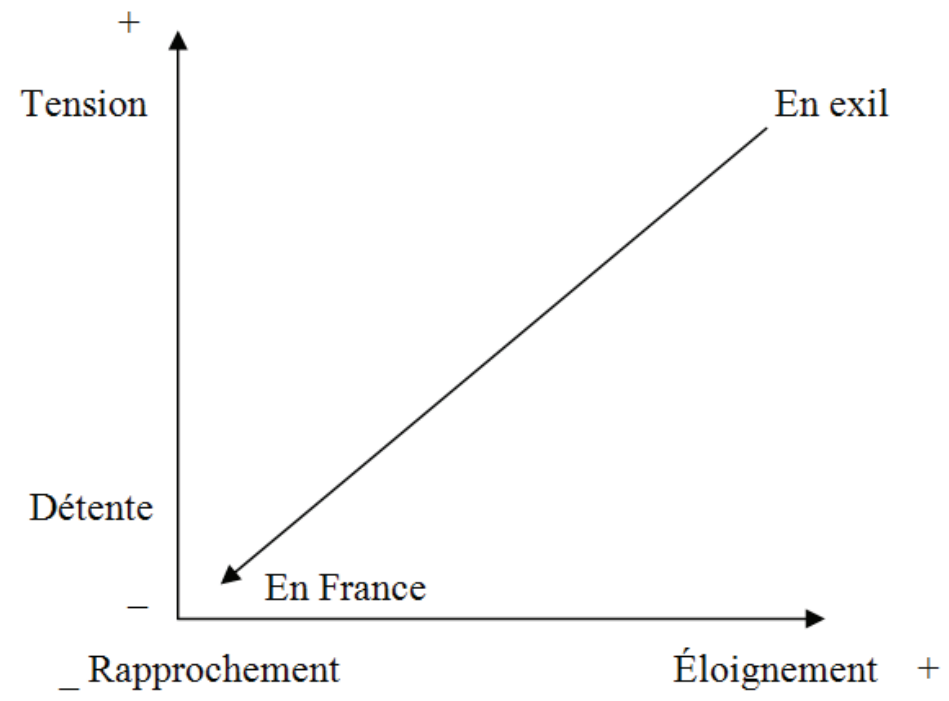

D'autre part, les allusions à l'importance de la nature 
- thème central dans l'œuvre hugolienne ${ }^{14}$ - sont très nombreuses : «je suis sous les branches des arbres », « je puis songer à la beauté des cieux », « je sens la paix de la grande nature / Qui m'entre dans le cœur», « assis au bord des ondes / Ému par ce superbe et tranquille horizon», « regarder les fleurs qui sont dans le gazon », " attendri par ces divins spectacles, / Plaines, forêts, rochers, vallons, fleuve argenté ». En effet, le corps propre du sujet fonctionne de façon normale, dans la mesure où il est capable d'homogénéiser l'extéroceptivité et l'intéroceptivité ${ }^{15}$ : l'univers extérieur est aussi paisible que l'univers intérieur.

Ce passage de la tension à la détente lui permet d'examiner en lui « les vérités profondes ». C'est-à-dire qu'il existe une relation converse entre «l'attendrissement par la nature » d'une part, et la reprise de la raison et l'examen des «vérités profondes » d'autre part.

Par ailleurs, le locuteur a appris qu'il est impossible de réduire Dieu à la position de l'anti-sujet, et du fait que Dieu est nécessairement un destinateur : il voit sa «petitesse » face aux « miracles » de Dieu. En réalité, il souligne une opposition modale fondamentale, entre un pouvoir faire maximal et un ne pas pouvoir ne pas faire.

14 Comme l'indique Louis Aguettant : « Le thème de la nature occupe une place centrale dans l'œuvre de Victor Hugo, car il se rattache de quelque façon à l'ensemble de ses idées maîtresses : tout changement dans les opinions ou dans la poétique de l'écrivain influe aussitôt sur sa conception de la nature, et même indirectement sur sa vision » (2000, p. 11, c'est l'auteur qui souligne) ; «Hugo a voulu créer en France une grande poésie de la nature. Il faut saluer son extraordinaire génie. Il a rendu avec une puissance neuve, incomparable, les tumultes de la mer, et les jeux de la lumière et de l'ombre dans le ciel. Et il a évoqué la figure du monde visible avec un éclat et une vigueur précises où la poésie française n'avait pas encore atteint » (2000, p. 460).

15 Selon la théorie sémiotique, c'est le corps propre qui permet l'homogénéisation du monde extérieur (l'extéroceptif) et du mon intérieur (l'intéroceptif), et qui assure donc l'accès à l'univers du sens. Voir par exemple : Coquet (1997, p. 08); Coquet (2007, p. 263) ; Greimas, Fontanille (1991, p. 324); Fontanille (2003, p. 250-251); Fontanille (2011, p. 11). 


\section{Réhabilitation de Dieu}

La prise de conscience de ces «vérités profondes » entraîne un changement radical dans l'horizon thymique et axiologique du sujet; autant dans "Trois ans après », il s'agissait de la péjoration de Dieu, autant il s'agit ici de son admiration :

Je viens à vous, Seigneur ! confessant que vous êtes

Bon, clément, indulgent et doux, ô Dieu vivant!

Je conviens que vous seul savez ce que vous faites,

Et que l'homme n'est rien qu'un jonc qui tremble au vent ;

Je dis que le tombeau qui sur les morts se ferme

Ouvre le firmament ;

Et que ce qu'ici-bas nous prenons pour le terme

Est le commencement ;

Je conviens à genoux que vous seul, père auguste, Possédez l'infini, le réel, l'absolu ;

Je conviens qu'il est bon, je conviens qu'il est juste

Que mon cœur ait saigné, puisque Dieu l'a voulu !

Je ne résiste plus à tout ce qui m'arrive

Par votre volonté.

L'âme de deuils en deuils, l'homme de rive en rive,

Roule à l'éternité.

Nous ne voyons jamais qu'un seul côté des choses ;

L'autre plonge en la nuit d'un mystère effrayant.

L'homme subit le joug sans connaître les causes.

Tout ce qu'il voit est court, inutile et fuyant (HUGO, 2008, p.

211-212).

Dans vos cieux, au delà de la sphère des nues,

Au fond de cet azur immobile et dormant, 
Peut-être faites-vous des choses inconnues

Où la douleur de l'homme entre comme élément.

Peut-être est-il utile à vos desseins sans nombre

Que des êtres charmants

S'en aillent, emportés par le tourbillon sombre

Des noirs événements (HUG0, 2008, p. 213).

On constate donc que Dieu est réhabilité : il est « [b] on », « clément », « indulgent », « doux »; ce Dieu n'est pas " jaloux », ni le « fatal maître », mais le " père auguste ». Un système d'oppositions entre les valeurs terrestres (humaines) et les valeurs célestes (divines) est établi : d'un côté tout ce qui est " court, inutile et fuyant », de l'autre, tout ce qui est « infini », « réel », « absolu » et « immobile ». Le sujet était attaché au premier type des valeurs, mais il s'efforce de voir le deuxième type, celui du destinateur. Il s'agit parfois des interprétations opposées du même fait selon que l'on accepte le premier ou le deuxième univers de valeurs : la « fin »vs le « commencement»; la «fermeture » vs l'« ouverture ». En d'autres termes, il s'agit d'un changement de point de vue : le sujet essaie de voir le monde du point de vue du destinateur, sans y réussir totalement; d'où la répétition de "peut-être ». Il rappelle que l'homme n'est capable de « voir qu'un seul côté des choses »; c'est « Dieu seul qui sait ce qu'il fait ». En effet, ce ne pas savoir n'est pas propre au sujet, il est valable pour tout être humain : le changement énonciatif au sein du premier passage (du « je » à « Nous » et à l'« homme ») le souligne.

Aussi le locuteur imagine-t-il la possibilité d'une interprétation positive des souffrances : peut-être «la douleur de l'homme », par exemple la disparition des êtres chers, entre-t-elle « comme élément» dans les programmes de Dieu. 
Cela nous conduit vers un schéma tensif, dans la mesure où l'unicité de l'interprétation des faits avait abouti à la colère et à la révolte, alors que la possibilité de sa multiplicité mène le sujet vers la soumission :

Schéma 4 - Interprétation et passion.

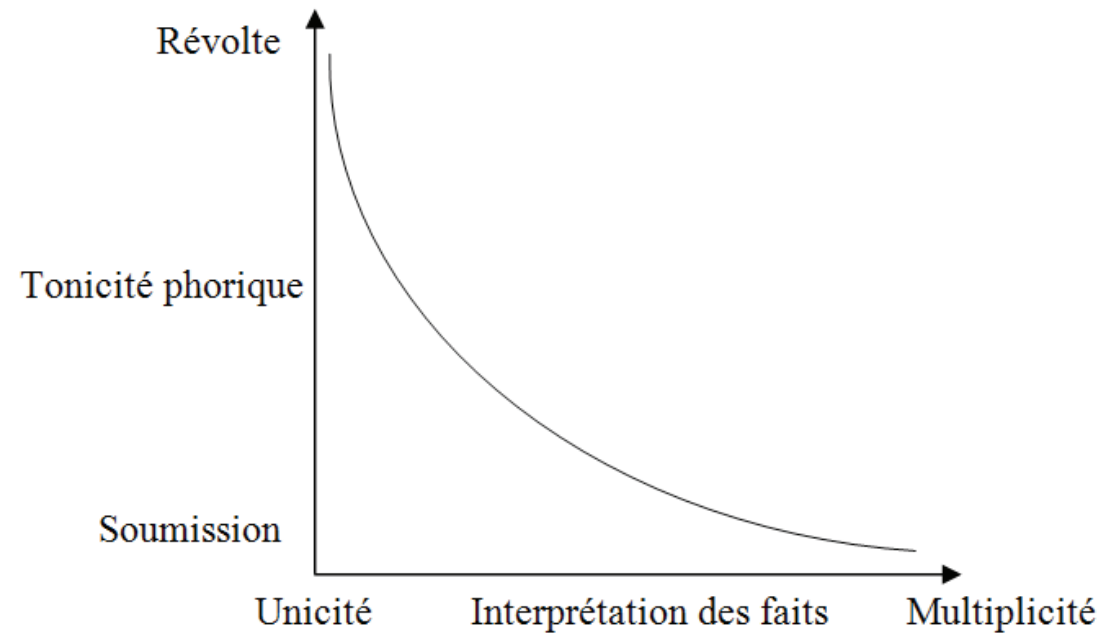

De même, le locuteur considère la sanction négative qu'il a subie comme «bon[ne] » et « juste », puisque c'est «Dieu [qui] l'a voulu[e] »: il ne « résiste plus » devant la «volonté » de Dieu. Plusieurs oppositions modales entre le sujet et le destinateur sont mentionnées : savoir vs ne pas savoir, pouvoir $v s$ ne pas pouvoir, vouloir $v s$ ne pas vouloir. Le sujet n'est pas capable de modifier les modalités ; la seule solution qu'il lui reste pour transformer son univers thymique, c'est d'accepter sa situation telle quelle, et de modifier ou au moins d'atténuer une seule de ses modalités : ne pas vouloir, ce qui n'est possible qu'avec un changement d'horizon axiologique. 
Aussi constate-t-on que le sujet, tout en rappelant ce qui s'est passé, revient vers Dieu, et même le supplie :

Je viens à vous, Seigneur, père auquel il faut croire ;

Je vous porte, apaisé,

Les morceaux de ce cœur tout plein de votre gloire

Que vous avez brisé ; [...] (HUGO, 2008, p. 211) ${ }^{16}$.

Je vous supplie, ô Dieu! de regarder mon âme,

Et de considérer

Qu'humble comme un enfant et doux comme une femme,

Je viens vous adorer! (HUGO, 2008, p. 213).

Maintenant que le sujet est en situation de détente (il est " apaisé »), il assume sa modestie, son infériorité et sa faiblesse : il est « humble comme un enfant et doux comme une femme », mieux, il « supplie». Il prend conscience qu'il ne peut pas ne pas croire dans son destinateur, et que ce croire même est modalisé par le devoir : "il faut croire ", même si le destinateur a été un anti-destinateur ; ce qui est tout à fait lié à la faiblesse du sujet et à l'absence de toute autre solution. Le régime dominant est donc d'ordre concessif, à savoir celui qui relie deux contenus avec pourtant (X pourtant $Y$ ), et qui correspond, de fait, à la surprise et à l'éclat ${ }^{17}$ : le destinateur a « brisé » les « morceaux » du « cœur » du sujet, pourtant le sujet revient vers lui pour l'adorer. Cela correspond à un vrai sujet soumis :

Ne vous irritez pas que je sois de la sorte,

16 Louis Veuillot note à propos de ce passage : «Il n'y a pas de plus beaux vers dans la langue française, ni dans la langue chrétienne » (cité par Journet et Robert, 1958, p. 126127).

17 Voir à ce sujet : Zilberberg (2012, p. 146 et p. 151). Le régime opposé est d'ordre implicatif : $\mathrm{X}$ donc $\mathrm{Y}$. 
Ô mon Dieu ! cette plaie a si longtemps saigné !

L'angoisse dans mon âme est toujours la plus forte,

Et mon cœur est soumis, mais n'est pas résigné (HUGO,

2008, p. 215).

Le sujet est « soumis », mais il «n'est pas résigné ». Une nuance significative est soulignée : si le sujet, par la force des choses, admet la rigueur divine, s'il accepte et assume le devoir qu'il subit, pourtant, le deuil reste toujours nonaccompli ; même s'il a « si longtemps » souffert, sa souffrance ne s'est même pas atténuée. Si le deuil était accompli, il ne souffrirait plus, il ne resterait plus dans l'interrogation de la volonté de Dieu. Il s'agit en quelque sorte d'une réapparition voilée de la révolte, entre la tension et la détente. Le sujet montre son mécontentement. La comparaison avec une strophe du premier poème du recueil serait intéressante, lorsque le locuteur s'adresse à sa fille :

Ô mon enfant, tu vois, je me soumets.

Fais comme moi : vis du monde éloignée ;

Heureuse ? non ; triomphante ? jamais.

- Résignée! (HUGO, 2008, p. 31).

Le locuteur conseille à sa fille d'être comme lui, d'être résignée. On constate donc que le sujet des Contemplations est en devenir : le sujet de «À ma fille » est un sujet soumis et résigné ${ }^{18}$; le sujet de « Trois ans après » n'est ni soumis, ni résigné; le sujet de «À Villequier » est soumis, sans être résigné. Le sujet détendu du début du recueil va progressivement

18 Ludmila Charles-Wurtz (2001, p. 77-78). affirme à propos de cette strophe : «Se résigner, c'est en effet accepter sans protester la volonté d'un supérieur (ici, celle de Dieu), mais aussi, si l'on se fie à l'étymologie, abandonner volontairement un droit. [...] si le père du poème «À ma fille» peut, par avance, prôner la résignation, c'est parce qu'il est métaphoriquement déjà mort ». 
jusqu'à la tension maximale, lors de la révolte, mais il se détend relativement, sans jamais revenir à cette détente initiale : un schéma d'amplification puissant est suivi d'un schéma d'atténuation moins fort ${ }^{19}$.

\section{Se justifier}

Par la suite, après avoir évoqué la sanction négative inattendue, suivie de son mécontentement, le sujet revient sur ses moments de colère et de révolte, en essayant de se justifier :

Qu'une âme ainsi frappée à se plaindre est sujette,

Que j'ai pu blasphémer,

Et vous jeter mes cris comme un enfant qui jette

Une pierre à la mer !

Considérez qu'on doute, ô mon Dieu ! quand on souffre, Que l'œil qui pleure trop finit par s'aveugler,

Qu'un être que son deuil plonge au plus noir du gouffre,

Quand il ne vous voit plus, ne peut vous contempler,

Et qu'il ne se peut pas que l'homme, lorsqu'il sombre

Dans les afflictions,

Ait présente à l'esprit la sérénité sombre

Des constellations!

Aujourd'hui, moi qui fus faible comme une mère,

Je me courbe à vos pieds devant vos cieux ouverts.

Je me sens éclairé dans ma douleur amère

Par un meilleur regard jeté sur l'univers.

Seigneur, je reconnais que l'homme est en délire,

19 Sur les schémas, voir Fontanille (2003, p. 111-112). 
S'il ose murmurer ;

Je cesse d'accuser, je cesse de maudire,

Mais laissez-moi pleurer !

Hélas! laissez les pleurs couler de ma paupière, Puisque vous avez fait les hommes pour cela! Laissez-moi me pencher sur cette froide pierre Et dire à mon enfant : Sens-tu que je suis là ? (HUGO, 2008, p. 214).

Ici, le locuteur a recours aux procédés rhétoriques significatifs : d'une part, en créant un espace intersubjectif, il s'adresse directement à Dieu ; d'autre part, il utilise systématiquement des termes qui permettent la généralisation, c'est-à-dire que tout être humain aurait fait la même chose que lui : "Considérez qu'on doute, ô mon Dieu ! quand on souffre », " une âme ainsi frappée », " un être que son deuil plonge au plus noir du gouffre », "l'homme est en délire », etc. (c'est nous qui soulignons). Il tente ainsi de déresponsabiliser, de ne pas assumer entièrement l'acte qu'il reconnaît pourtant avoir accompli : «j'ai pu blasphémer». De plus, avec le verbe «Considérez », répété aussi dans d'autres strophes du poème, le locuteur invite Dieu à adopter son point de vue, et à lui donner raison.

Par ailleurs, un schéma tensif est évoqué : d'un côté, c'est la dysphorie qui s'installe; de l'autre, c'est la contemplation (« L'œil qui pleure trop finit par s'aveugler [...] il ne vous voit plus, ne peut vous contempler »). La relation entre les deux axes est donc inverse : 
Schéma 5 - Justification de la colère.

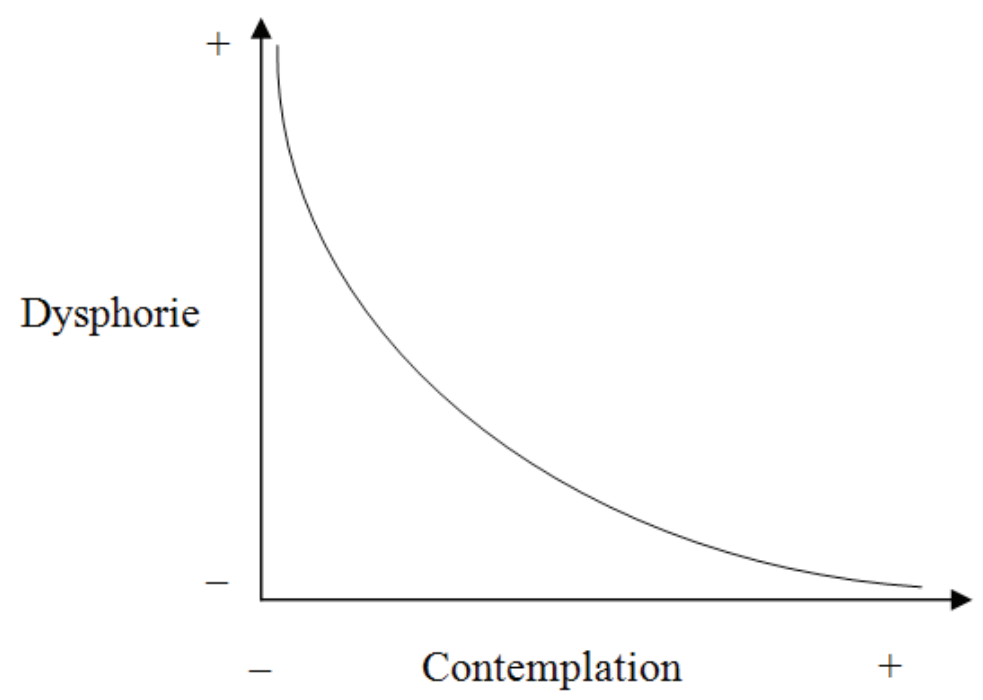

La dysphoriea ététellementintense quela contemplation est devenue trop réduite, voire nulle. Il s'agit du rappel de la transformation du sujet en non-sujet lors de la révolte : un sujet ému, agité et aveugle est un sujet privé de cognition, voire de perception.

Lorsque le locuteur affirme que l'être humain, aux moments des «afflictions $»^{20}$, ne peut pas avoir présent à l'esprit « la sérénité » des « constellations », il reformule le même schéma. Cette relation inverse est modalisée par le verbe impersonnel «il ne se peut pas », autrement dit par le devoir être.

Mais, comment est-il possible que le sujet dont la souffrance est restée aussi «forte », arrive à « reprendre sa

20 Rappelons que l'affliction, selon l'assertion de Hugo (1985 [1866], p. 302) lui-même dans Les Travailleurs de la mer, c'est le troisième degré de désespoir : «Le désespoir a des degrés remontants. De l'accablement on monte à l'abattement, de l'abattement à l'affliction, de l'affliction à la mélancolie ». 
raison » et à « contempler » ? Comment est-il possible qu'il se sente « éclairé » malgré sa " douleur amère », et qu'il puisse jeter « un meilleur regard [...] sur l'univers » ? C'est qu'il a été jusqu'à présent dans le régime implicatif (rupture du contrat fiduciaire donc mécontentement), mais actuellement, il adopte une logique concessive (rupture du contrat fiduciaire pourtant contentement) : sa révolte est d'ordre implicatif, alors que sa soumission est d'ordre concessif.

Avec le syntagme "moi qui fus faible », d'une part le locuteur assume à présent qu'il était «faible » au moment de la révolte, lorsqu'il s'était laissé emporter par le régime implicatif ; d'autre part, en utilisant le passé simple, il montre sa différence radicale avec cet état-là, comme s'il était maintenant fort! On en prend pour preuve l'annonce catégorique de la révolte : "Je cesse d'accuser, je cesse de maudire », et il formule tout de suite des demandes : " Mais laissez-moi pleurer », "Laissez-moi me pencher sur cette froide pierre »; c'est-à-dire que la faiblesse émerge sous un nouveau jour : d'une part, il reconnaît que son acte de révolte était comme l'acte d'« un enfant qui jette / Une pierre à la mer »; de l'autre, il s'assujettit : « Je me courbe à vos pieds », «vous [Dieu] avez fait les hommes pour cela [= pleurer]».

En d'autres termes, si au moment de la colère, il avait opté pour le paradigme de la paternité au détriment du paradigme de la divinité, maintenant il se rend compte que le premier paradigme ne peut se définir que sous le contrôle du deuxième : même pour pleurer sur la tombe de sa fille, il doit prier auprès de Dieu.

En bref, après la tension due à la colère, suite au changement de situation dans sa vie et à la modification de son univers axiologique, le locuteur se trouve dans la situation assez détendue de la soumission, et en réhabilitant Dieu, se justifie auprès de lui. 


\section{Pour finir}

Jacques Fontanille postule l'existence d'un " espace » sous-jacent à tout discours, « traversé par des énergies et qui prendrait sens, entre autres, quand les articulations narratives et discursives viendraient y projeter des seuils, des frontières, des zones d'accumulation ou de raréfaction de l'énergie » (FONTANILLE, 1993, p. 15). Ainsi la séquence canonique de la colère, présupposant « la tension d'une attente, que la disjonction (la frustration) va transformer en accumulation d'énergie ", se clôt-elle par une explosion, qui implique " une libération de l'énergie accumulée, pour parvenir à une détente » (FONTANILLE, 1993, p. 15). La soumission, elle, ne réclamant pas d'attente à proprement parler, implique un dégagement d'énergie, et donc la détente. En effet, de façon générale, la colère suppose une intensité forte et une extensité temporelle réduite; en revanche, la soumission suppose une intensité faible et une extensité temporelle diffuse :

Schéma 6 - Intensité et extensité passionelles.

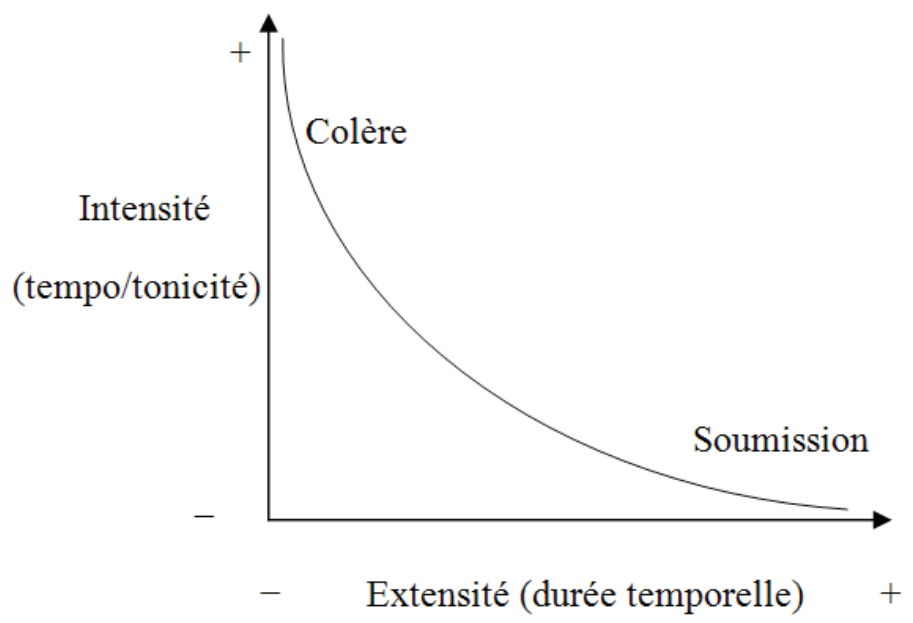


C'est dire qu'il s'agit d'un côté de l'éclat tonique et de l'accélération, de l'autre, de l'atonie et de la lenteur. En termes aspectuels, la colère est définie par l'aspect ponctuel, par son caractère explosif, foudroyant, paroxystique ${ }^{21}$, alors que la soumission est caractérisée par l'aspect duratif, par l'itération du même contenu passionnel. Si la colère implique un sujet dont la dimension passionnelle l'emporte sur la dimension cognitive, et qui tend vers le non-sujet, la soumission correspond à un sujet cognitivo-passionnel plutôt stable.

Par ailleurs, la soumission et la colère, du moins dans le cas ici étudié, semblent être deux façons différentes de manifester son impuissance face à la puissance du destinateur : peut-être le sujet soumis est-il celui qui assume son impuissance mieux que le sujet de colère ; le sujet soumis reconnaît la domination du devoir sur le vouloir sans aucun effort pour changer sa situation, alors que l'autre fait une tentative illusoire pour inverser la domination, c'est dire que la colère ne peut être que l'« espoir de soulagement » (PARRET, 1986, p. 111) 22 : « l'explosion de la colère ne résout rien d'autre que le malaise du sujet » (FONTANILLE, 2005, p. 65) ${ }^{23}$.

\section{RÉFÉRENCES}

AGUETTANT, L. Victor Hugo poète de la nature. Paris : L'Harmattan, 2000.

21 Jean-Claude Anscombre dit que la colère est une passion « externe provisoire » (1996, p. 268). D'autre part, Herman Parret, en commentant Thomas d'Aquin, indique : « La colère qui persiste engendre la haine » (1986, p. 112, c'est l'auteur qui souligne).

22 Il explique la conception d'Alain.

23 Il reprend l'idée de Sénèque. 
ANSCOMBRE, J.-C. " Noms de sentiment, noms d'attitude et noms abstraits ». FLAUX, N. ; GLATIGNY, M. ; SAMAIN, D. (Org.). Les Noms abstraits. Histoire et théories. Villeneuved'Ascq : Presses Universitaires du Septentrion, 1996. p. 257273.

BERTRAND, D. Précis de sémiotique littéraire. Paris : Nathan, 2000.

BOREL, P. Rhapsodies. Thurins : Fougerouse, 2009 [1832].

CHARLES-WURTZ, L. Les Contemplations de Victor Hugo. Paris : Gallimard, 2001.

Poétique du sujet lyrique dans l'œuvre de Victor Hugo. Paris : Honoré Champion, 1998.

COLLOT, M. La Matière-émotion. Paris : Presses Universitaires de France, 1997.

COQUET, J.-C. Phusis et Logos : une phénoménologie du langage. Saint-Denis : Presses Universitaires de Vincennes, 2007.

. Le Discours et son sujet. Paris : Méridiens Klincksieck, 1989 [1984].

La Quête du sens : le langage en question. Paris : Presses Universitaires de France, 1997.

FONTANILLE, J. Corps et sens. Paris : Presses Universitaires de France, 2011.

. « Colère ». In : DITCHE, E. R. ; FONTANILLE, J. ; LOMBARDO, P. Dictionnaire des passions littéraires. Paris : Belin, 2005. p. 61-79.

- Sémiotique du discours. Limoges : Presses Universitaires de Limoges, 2003. 
- "Le désespoir ou les Malheurs du cœur et le salut de l'esprit ». Actes sémiotiques-documents, Paris, t. II, n. 16, 1980.

. "L'émotion et le discours ». Sémiotique de l'affect. Protée, Québec, v. 21, n. 2, p. 13-19, 1993.

GREIMAS, A. J. ; FONTANILLE, J. Sémiotique des passions : des états de choses aux états d'âme. Paris : Seuil, 1991.

HUGO, V. Les Contemplations. Paris : Flammarion, 2008 [1995].

Les Misérables. t. 2. Paris : Librairie Générale Française, 1998 [1862].

. Les Travailleurs de la mer. In : . OEuvres complètes. Roman. t. III. Paris : Robert Laffont, 1985 [1866]. p. 43-343.

Les Châtiments. In : OEuvres poétiques. t. 2 . Paris : Gallimard, 1967 [1853]. p. 01-247.

JOURNET, R. ; ROBERT, G. Notes sur « Les Contemplations ». ANNALES LITTERAIRES DE L'UNIVERSITE DE BESANÇON. Paris : Les Belles Lettres, 1958. v. 21.

MUSSET, A. La Coupe et les lèvres. Spectacle dans un fauteuil. Clermont-Ferrand : Paleo, 2007 [1831]. p. 07-88.

PARRET, H. Les Passions : essais sur la mise en discours de la subjectivité. Liège-Bruxelles : Mardaga. 1986.

RALLO DITCHE, É. ; FONTANILLE, J. «Introduction ». In : DITCHE, E. R. ; FONTANILLE, J. ; LOMBARDO, P. Dictionnaire des passions littéraires. Paris : Belin, 2005. p. 05-15.

VIGNY, A. Poèmes antiques et modernes. Clermont-Ferrand : Paleo, 2007 [1826]. 
ZILBERBERG, C. La Structure tensive. Liège : Presses Universitaires de Liège, 2012.

Cheminements du poème : Baudelaire, Rimbaud, Valéry, Jouve. Limoges : Lambert-Lucas, 2010.

Éléments de grammaire tensive. Limoges : PULIM, 2006.

Artigo recebido em setembro de 2014 e aprovado em dezembro de 2014.

Disponível em: http://seer.fclar.unesp.br/casa 